\title{
A HYBRID MODEL FOR STUDYING SPATIAL ASPECTS OF INFECTIOUS DISEASES
}

\author{
BENJAMIN J. BINDER ${ }^{\otimes} 1$, JOSHUA V. ROSS ${ }^{1}$ and MATTHEW J. SIMPSON ${ }^{2}$
}

(Received 25 May, 2012; revised 4 September, 2012; first published online 7 February, 2013)

\begin{abstract}
We consider a hybrid model, created by coupling a continuum and an agent-based model of infectious disease. The framework of the hybrid model provides a mechanism to study the spread of infection at both the individual and population levels. This approach captures the stochastic spatial heterogeneity at the individual level, which is directly related to deterministic population level properties. This facilitates the study of spatial aspects of the epidemic process. A spatial analysis, involving counting the number of infectious agents in equally sized bins, reveals when the spatial domain is nonhomogeneous.
\end{abstract}

2010 Mathematics subject classification: primary 37B15; secondary 92D30.

Keywords and phrases: agent-based model, continuum model, infectious diseases, spatial heterogeneity.

\section{Introduction}

There exist a variety of approaches to the mathematical modelling of infectious diseases, with deterministic, stochastic and agent-based methods all having been used to study the spread of infection in a population $[2,3,8,18,20,21,24,25,31,33]$. The majority of these models are ordinary differential equations (that is, continuum) or Markov chain models, and ignore the spatial aspects of the epidemic process. The benefit of these models is that they are typically relatively easy to analyse and to parameterize using data.

More recent work has investigated the use of agent-based models to describe the localized spatial properties of an epidemic [33]. The classic work of Dr. John Snow on cholera in London is an excellent example to highlight the importance of studying the spatial aspects of disease spread in order to facilitate control [30]. The importance of spatial effects in controlling disease spread has been relevant in Australia. For

\footnotetext{
${ }^{1}$ School of Mathematical Sciences, University of Adelaide, South Australia 5005, Australia; e-mail: benjamin.binder@adelaide.edu.au, joshua.ross@ adelaide.edu.au.

${ }^{2}$ Mathematical Sciences School, Queensland University of Technology, Brisbane, Queensland 4001, Australia; e-mail: matthew.simpson@qut.edu.au.

(c) Australian Mathematical Society 2013, Serial-fee code 1446-1811/2013 \$16.00
} 
example, the first confirmed case in Australia of the 2009 influenza A(H1N1)pdm pandemic was recorded in Queensland from a passenger who arrived from the United States on May 9 [22]. Victoria confirmed its first case on May 30, and Western Australia confirmed its first case on May 24 [22]. In this context, it makes little sense to treat the entire Australian population as a well-mixed system since spatial effects were important in the way that the disease was introduced into Australia. In this particular example, these spatial differences seem to be very important as the disease progression in Victoria and Western Australia was very different. Spatial effects could also be very important in the way that different Australian states (or different regions within each state) respond in terms of introducing different public health strategies at various times during an epidemic. Agent-based models provide one avenue for investigating these important spatial differences. However, agent-based models come with difficulties in terms of the choice of rules governing dynamics and the intimately related parameterization using data.

The term hybrid modelling (continuum and agent-based) has several meanings in the existing literature [15], and perhaps the most common use is to describe a system in which some of the quantities of interest are modelled as a continuum while other quantities are modelled as collections of agents [1, 10, 11, 16, 17]. Alternatively, hybrid modelling can refer to using both the continuum and agent-based descriptions for the same quantity of interest [8, 14, 26, 32]; the idea behind this approach is to switch from the agent-based to the continuum description when the number of agents reaches some threshold within spatial regions of the domain, typically dictated by computational feasibility.

Here we consider a hybrid model which is a first attempt to develop a framework for infectious disease modelling that allows for the study of the spatial properties of an epidemic, yet is still relatively easy to parameterize. Our model is unlike other agent-based models, where the numbers of agents and events evolve directly from the agent-based mechanisms [4, 7, 27, 29]. Our approach, on the other hand, allows for precise modelling of the spatial aspects under prescribed population level changes. To demonstrate the potential of this framework, we perform a spatial analysis to reveal when the spatial domain is nonhomogeneous.

The hybrid model consists of transmission and motility mechanisms. There is no mechanism for the recovery or removal of infected individuals. The (agent-based) mechanisms are similar to the cell proliferation and motility rules used in earlier work by the first and third authors [4, 7, 27, 29]. Only nearest neighbour events are considered, but other long distance events such as next nearest neighbour transmissions could be modelled using our framework. We first consider these mechanisms separately before combining them in our complete hybrid model of an epidemic.

\section{Transmission}

Consider a population which consists of susceptible, infectious and recovered (or removed) individuals. The so-called S IR model [12] describes the evolution of the 
proportions $S(t), I(t)$ and $R(t)$, respectively, of these three types, with transmission $\beta$ and recovery $\gamma$ rate parameters; the model consists of the system of ordinary differential equations

$$
\begin{aligned}
& \frac{d S(t)}{d t}=-\beta S(t) I(t), \\
& \frac{d I(t)}{d t}=\beta S(t) I(t)-\gamma I(t), \\
& \frac{d R(t)}{d t}=\gamma I(t) \\
& S(t)+I(t)+R(t)=1 .
\end{aligned}
$$

When there is no recovery of infectious individuals $(\gamma=0)$, the $S I R$ model reduces to the $S I$ model (with $R(0)=0$ ). Using (2.1), (2.2) and (2.3), the evolution of the proportion of infectious individuals in the population is then governed by the single ordinary differential equation

$$
\frac{d I(t)}{d t}=\beta I(t)(1-I(t)),
$$

with solution

$$
I(t)=\frac{I(0) e^{\beta t}}{1+I(0)\left(e^{\beta t}-1\right)} .
$$

If the spread of infection is due solely to the transmission mechanism, equation (2.4) is the basis of the continuum component of our hybrid model, which is subsequently coupled to an agent-based model.

The agent-based component of the hybrid model consists of a rectangular lattice domain with agents located at the discrete integer points $(x, y)$, where $1 \leq x \leq L$ and $1 \leq y \leq H$. Each lattice site is occupied by either a single infectious or susceptible agent. The total number of infectious agents $n(t)$ at any discrete time $t$ is predetermined by (2.4), and we have

$$
n(t)=\lfloor I(t) L H\rfloor .
$$

During a single time step from $t$ to $t+1$, infectious agents are selected using the random sequential update method described by Chowdhury et al. [9] and given the opportunity to transmit the infection to susceptible agents. A chosen infectious agent at $(x, y)$ attempts to transmit the infection to one of the nearest neighbour sites $(x \pm 1, y \pm 1)$, with equal probability $1 / 4$. If the target site for transmission is occupied by a susceptible agent, the susceptible agent changes to an infectious agent; see Figure 1(a). If the target site for transmission is already an infectious agent, the event is aborted. This process is repeated until the total number of infectious agents in the domain is $n(t+1)$.

Shown in Figures 2(b) and 2(c) are simulations of the spread of infection for a transmission rate parameter $\beta=0.125$ and $I(0)=0.1$. The domain was initially 
(a) Transmission rule

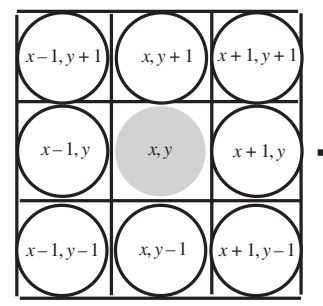

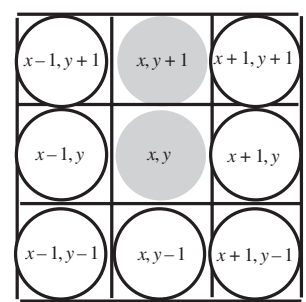

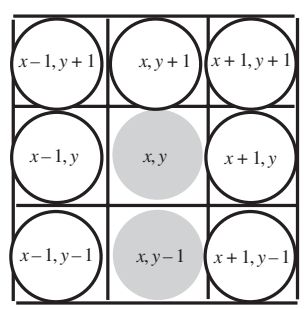

(b) Motility rule

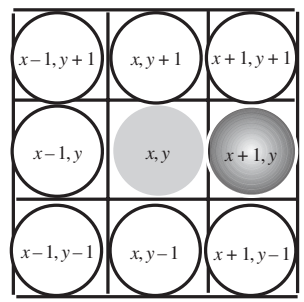

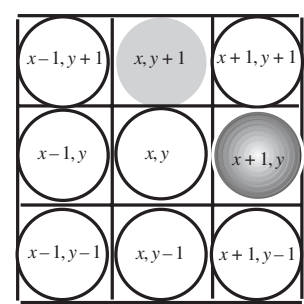

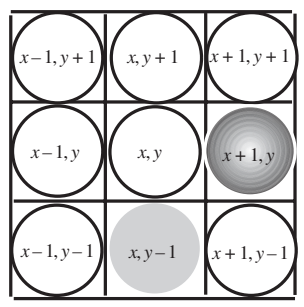

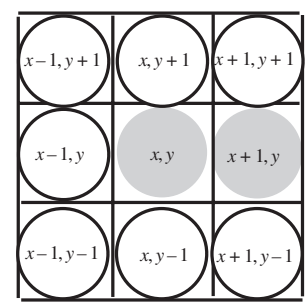
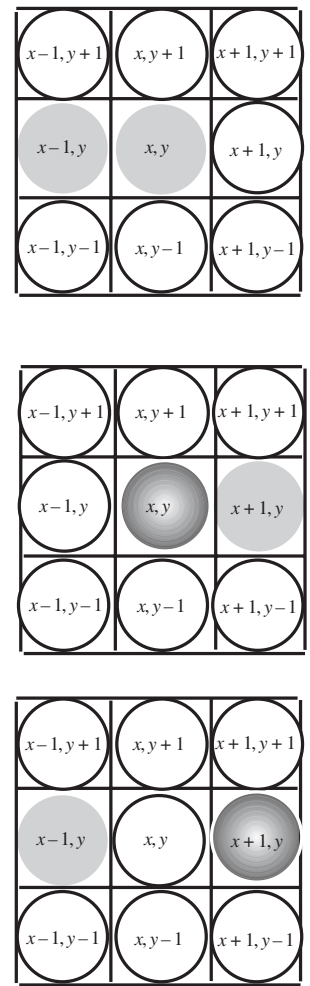

FIGURE 1. Agent transmission and motility mechanisms, susceptible (white) and infectious (light and dark grey).

populated uniformly at random with $n(0)=I(0) L H$ infectious agents; see Figure 2(a). A check that the evolution of the proportion of infectious agents within the domain is the same as that of (2.4) is illustrated in Figure 2(d). This is expected, as the agent numbers are predetermined by the continuum component of the hybrid model. The results show that there is spatial dependence in the domain, forming clusters of infectious hosts. This spatial heterogeneity is also observed for a nonuniform initial condition, as illustrated in Figures 2(e) and 2(f).

Next we consider solely the motility mechanism, before combining both the transmission and motility mechanisms in the hybrid model. 

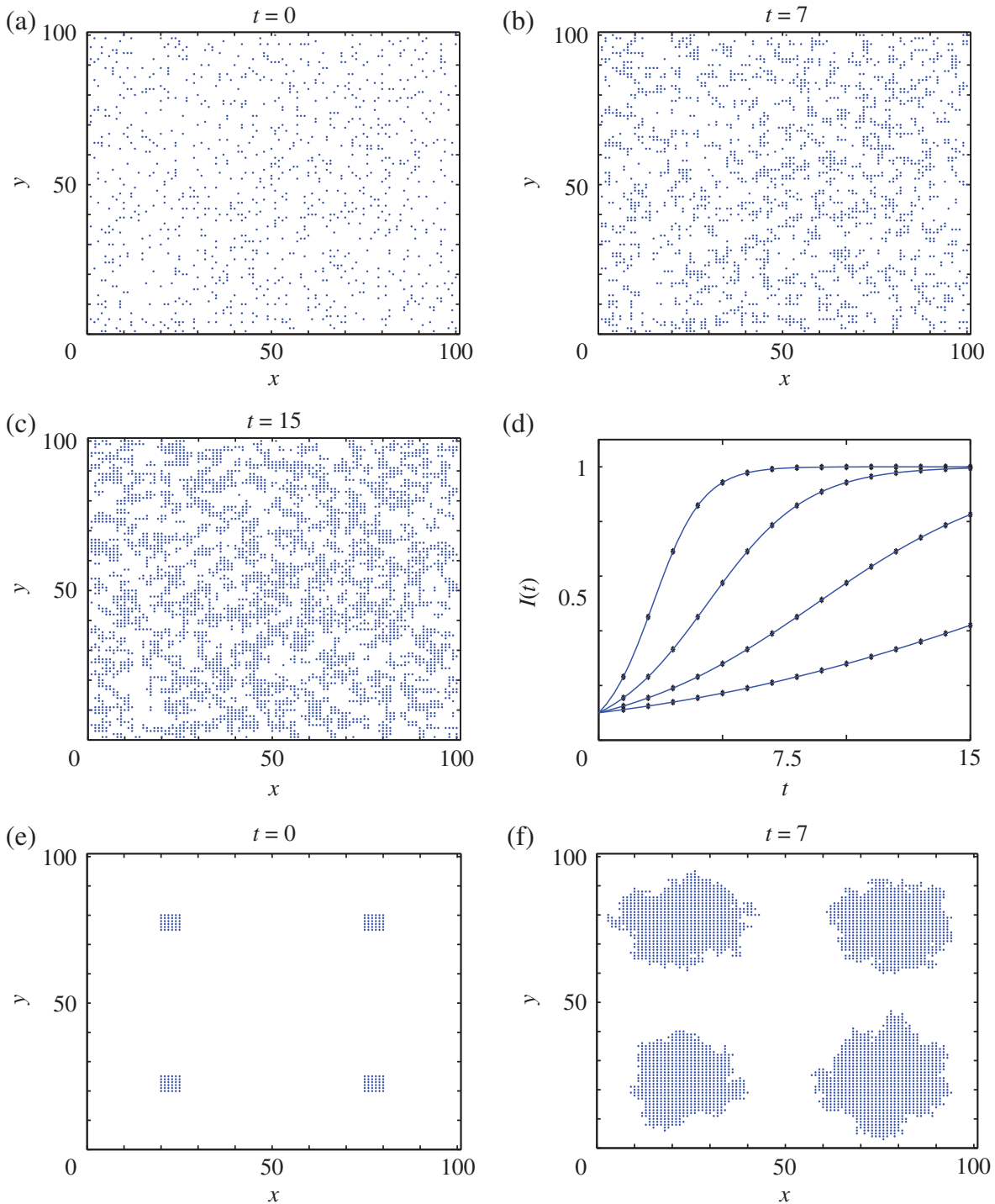

FIGURE 2. Transmission of infection for $L=H=100$. (a)-(c) Simulations with $I(0)=0.1$ and $\beta=0.125$. (d) Evolution of the proportion of the population that is infectious for $I(0)=0.1$. The markers are from the hybrid model and the solid curves are solutions of (2.4). From bottom to top, $\beta=0.125,0.25,0.5,1$. (e) and (f) Simulations with $I(0)=0.015$ and $\beta=0.5$.

\section{Motility}

The continuum component of the hybrid model is now spatially dependent, and the evolution (in the $x$ direction) of the proportion of infectious individuals within the population is $I(x, t)$. Assuming that the infectious hosts undergo an unbiased random 
walk, we have the linear diffusion equation

$$
\frac{\partial I(x, t)}{\partial t}=D \frac{\partial^{2} I(x, t)}{\partial x^{2}}
$$

where $D$ is the diffusivity coefficient.

When there is no transmission of infection, the total number of agents remains constant for all time. For the agent-based component of the hybrid model, at any discrete time $t$ there are

$$
n=\left\lfloor H \int_{0}^{L} I(x, 0) d x\right\rfloor
$$

infectious agents in the domain. The predetermined total number of agent-based motility events during a time step is related to the diffusivity by

$$
n_{d}=\lfloor 4 D n\rfloor .
$$

For each agent-based motility event, an infectious agent is selected using the random sequential update method described by Chowdhury et al. [9] and given the opportunity to move. A selected infectious agent at $(x, y)$ moves to one of the nearest neighbour sites $(x \pm 1, y \pm 1)$, each having equal probability $1 / 4$. The agent (susceptible or infectious) residing at the target site swaps its position with the infectious agent at $(x, y)$; see Figure 1(b). This consequently results in an unbiased random walk; the movement probabilities are unaffected by the proportion of infectious and susceptible agents within the total population.

Figure 3(a) is a typical initial condition of the hybrid model. The columns of the lattice, $c(x, 0)$, were populated uniformly at random with infectious agents according to

$$
c(x, 0)=\frac{H}{2}(\Theta(x-40)-\Theta(x-60)),
$$

where $\Theta(x)$ is the Heaviside or unit step function. A simulation of the initial condition is shown in Figure 3(b).

A realization of the path taken by an infectious agent $(x(t), y(t))$ while on its random walk is presented in Figure 3(c). The evolution of the squared displacements of this (tracked) infectious agent is given by

$$
X(t)=\sum_{i=1}^{t}(x(i)-x(i-1))^{2}, \quad Y(t)=\sum_{i=1}^{t}(y(i)-y(i-1))^{2},
$$

which is illustrated in Figure 3(d). As expected, the gradients $X^{\prime}(t)$ and $Y^{\prime}(t)$ are related to the diffusivity by

$$
X^{\prime}(t) \approx Y^{\prime}(t) \approx 2 D
$$

This provides a check that the hybrid model is consistent with the continuum model (3.1). 
(a)

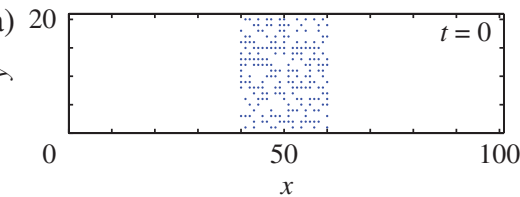

(c)
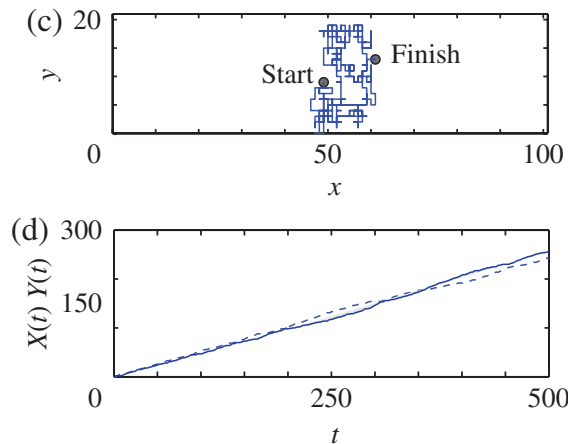

(b)

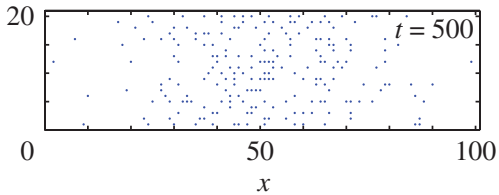

(e)

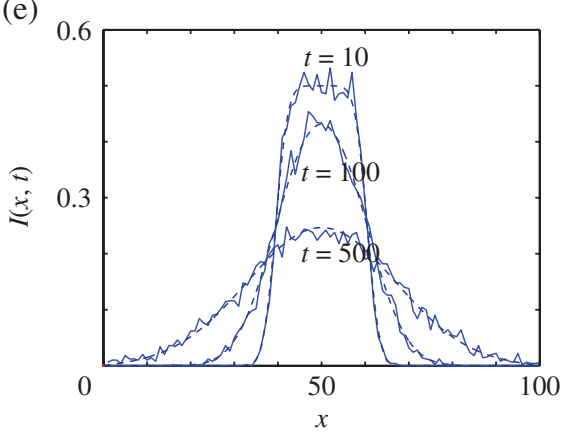

FIgure 3. Motility of infection for $D=0.25, L=100$ and $H=20$. (a) Typical initial condition for infectious agents. (b) Simulation of the initial condition, using the hybrid motility mechanism. (c) Typical path taken by an infectious agent during its random walk. (d) Squared displacement of path taken by an infectious agent. The solid curve is $X(t)$ and the dashed curve $Y(t)$. (e) Proportion of infectious agents (solid curves, $N=25$ ) from the hybrid model compared with the solution (dashed curves) of the linear diffusion equation (3.1).

Another comparison between the hybrid (solid curves) and continuum (dashed curves) models is made in Figure 3(e). For this comparison, we first define $c_{i}(x, t)$ to be the total number of infectious agents in column $x$ of the lattice after $t$ steps of the $i$ th realization. Then for $N$ realizations starting from the same initial condition, we can define an average column infectious agent proportion as

$$
I_{h}(x, t)=\frac{1}{N Y} \sum_{i=1}^{N} c_{i}(x, t)
$$

which is compared to the solution of the linear diffusion equation (3.1). We see that there is excellent agreement between the hybrid and continuum models in Figure 3(e).

Next we consider both the transmission and motility mechanisms in the hybrid model.

\section{Transmission and motility}

When the transmission and motility mechanisms are combined, the corresponding continuum model is Fisher's equation,

$$
\frac{\partial I(x, t)}{\partial t}=D \frac{\partial^{2} I(x, t)}{\partial x^{2}}+\beta I(x, t)(1-I(x, t)) .
$$



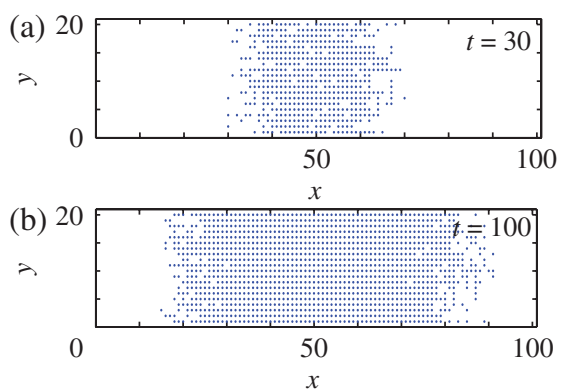

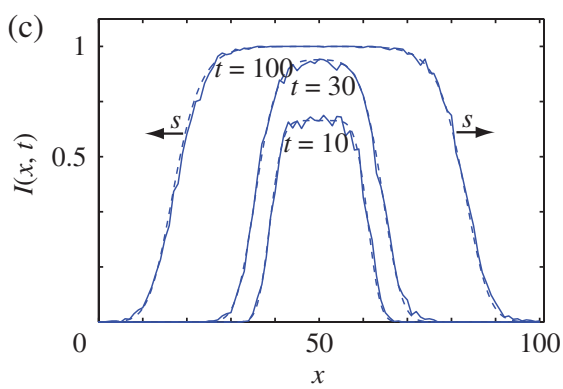

FIgure 4. Transmission and motility of infection for $D=0.25, \beta=0.1, L=100$ and $H=20$. (a) and (b) Simulations of the initial condition shown in Figure 3(a). (c) Proportion of infectious agents (solid curves, $N=25$ ) from the hybrid model compared with the solution of Fisher's equation (dashed curves).

Now both the transmission and motility rules are applied to the infectious agents in the domain. The predetermined number $n(t)$ of infectious agents in the hybrid model at any time $t$ is given by the solution to Fisher's equation and

$$
n(t)=\left\lfloor H \int_{0}^{L} I(x, t) d x\right\rfloor .
$$

The integral is solved numerically using the trapezoidal rule. Equation (4.1) is solved using a finite difference scheme with constant grid spacing $\delta x$ and implicit Euler stepping with constant time steps $\delta t$. A Picard linearization, with tolerance $\epsilon$, is used to solve the transformed nonlinear algebraic equations. All results presented in this work correspond to $\delta x=0.1, \delta t=0.05$ and $\epsilon=1 \times 10^{-4}$. The number of motility events during a time step of the hybrid model is then

$$
n_{d}(t)=\lfloor 4 D n(t)\rfloor
$$

The results in Figure 4(c) show that the average properties of the hybrid model (3.2) follow the solution of Fisher's equation (4.1). After sufficient time, for this initial condition we see two travelling waves of infection with constant speed

$$
s=2 \sqrt{\beta D},
$$

moving in both the negative and positive $x$ directions. Following Simpson et al. [28], we checked that our simulations matched this result for the wave speed.

Next we illustrate the potential for using this framework to study the spatial properties of the epidemic process, by investigating the evolution of the spatial distribution of an initial population of infectious agents that is dispersed uniformly at random throughout the whole domain.

\section{Spatial analysis}

The spatial distribution of the infectious agents may be quantified by calculating a scaled variance or index $[5,6,23]$. To calculate the index, the domain $L H$ is divided 
into $M$ equal-sized square bins. At any time $t$ there are a total of $n(t)$ infectious agents in the domain $L H$. If $b_{i}(t)$ is the number of infectious agents in bin $i, i=1, \ldots, M$, then the average bin count is $n(t) / M$ and the variance of the bin counts is

$$
\sigma^{2}(t)=\frac{1}{M} \sum_{i=1}^{M}\left(b_{i}(t)-\frac{n(t)}{M}\right)^{2} .
$$

The index is a scaled variance given by

$$
j(t)=\frac{\sigma^{2}(t)}{\sigma_{0}^{2}(t)},
$$

where

$$
\sigma_{0}^{2}(t)=n^{2}(t)\left(\frac{M-1}{M^{2}}\right)
$$

The index (5.1) ranges from unity, for a completely segregated state (when all infectious agents are in just one bin), to zero, for an evenly distributed state (when each bin contains precisely the average bin count). Although the evenly distributed state is possible, it is not often realized. Instead, a more realistic situation occurs when each infectious agent is equally likely to reside in any bin. This is known in the literature as the complete spatial randomness (CSR) state [13, 19, 23]. The CSR limit for the index $[5,6]$ is

$$
J_{\mathrm{CSR}}(t) \approx \frac{1}{n(t)}-\frac{1}{L H}
$$

We compare this limiting value to the average index, defined as

$$
J(t)=\frac{1}{N} \sum_{i=1}^{N} j_{i}(t)
$$

where $j_{i}(t)$ is the $i$ th realization of the index.

Presented in Figure 5 are results for identically prepared initial conditions $c(x, 0)=$ $0.1 H$ of infectious agents which are distributed uniformly at random throughout the entire spatial domain. When visually inspecting the simulations in Figures 5(a) and 5(b), for two values of the transmission rate, we see that it is difficult to ascertain whether the infectious agents are distributed at random throughout the domain. The evolution of the average index for these two values of the transmission rate is compared to the CSR values in Figure 5(d). For the lower value of the transmission rate parameter, $\beta=0.01$, the spatial domain of infectious agents is well homogenized as the average index is close to the CSR limit. This contrasts with the higher value of the transmission rate parameter, $\beta=0.2$, where the spatial distribution is nonhomogeneous, as the average index deviates from the CSR limit. Only when $t=0$ (that is, the initial condition) and when the lattice is completely occupied with infectious agents is the CSR state achieved. 
(a)
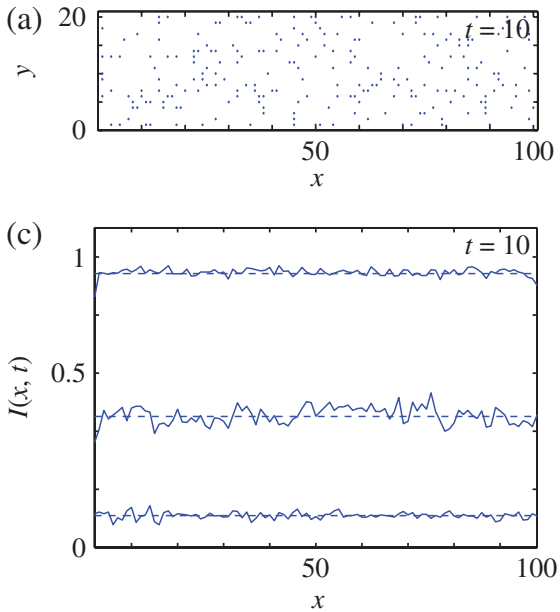

(b)
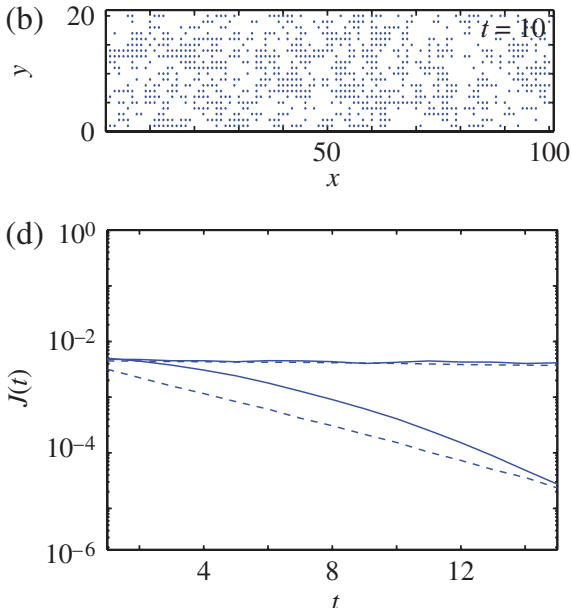

FIgure 5. Spatial analysis with $D=0.25, c(x, 0)=0.1 H, L=100$ and $H=20$. (a) Simulation with $\beta=0.01$. (b) Simulation with $\beta=0.2$. (c) Proportion of infectious agents (solid curves, $N=25$ ) from the hybrid model compared with the solution of Fisher's equation (dashed curves). From bottom to top, $\beta=0.01,0.2,0.5$. (d) Average index with $M=80$ equal-sized bins (solid curves, $N=25$ ) and CSR limiting value (dashed curves). From bottom to top, $\beta=0.2,0.01$.

\section{Discussion}

We have described a hybrid model for the spread of an infection in a closed population of susceptible and infectious individuals. The hybrid model's average properties are coupled to a continuum model. This provides a useful framework to investigate the spatial properties of the system at both the individual and population levels.

The spatial aspects of an epidemic are often of much interest, and vital to effective control, yet parameterizing and studying spatial models for subsequent use in control scenario testing is extremely difficult. Here we have linked the agent-based model with average properties of the system through a continuum model. This allows for partial parameterization via matching the (average) population level properties to data, and then subsequently considering the spatial aspects. It allows for assessing nonhomogeneity in the epidemic process, which may lead to the identification of mechanisms for more effective control. Moreover, if highly detailed population level data of an epidemic exist, then the continuum component of our model could be replaced with the empirical growth rate, reducing the dimension of the system by exactly matching the population level properties of the epidemic.

The agent-based model considered here could be extended further. For example, geographical barriers to transmission, and other spatial heterogeneities in the landscape occupied by the population, may be captured by changing the probabilities of movement; this then allows for a study of the spatial patterns that arise for a given (average) population level growth in an epidemic in a given environment. 
These (average) spatial patterns may be used to identify possibly novel behaviours in host movement and transmission, by comparison to completely at-random results; furthermore, they may be used for parameterizing from spatial data.

To illustrate the potential of this framework for studying the spatial aspects of an epidemic, we investigated the evolution of the spatial distribution of an initial population of infectious agents that is dispersed uniformly at random. The spatial index considered provides a summary of the spatial heterogeneity in a domain, but there is much room for extending this analysis. Ideally, we wish to develop a hypothesis test that allows us to test whether the infectious agents are uniformly distributed in the domain or not. Our framework allows us to develop such a test, and one in which we can hold the population level properties constant.

Finally, our concept of hybrid modelling could be extended to study more complex systems in epidemiology. For example, introducing a subpopulation of recovered individuals (the SIR model) with motility would lead to a system of partial differential equations, which would be coupled to the agent-based modelling. Future work will consider all of these extensions.

\section{Acknowledgements}

We thank Axel Ahmer for his assistance with the research. The contribution of Joshua Ross was funded by the Australian Research Council's Discovery Project funding scheme, project number DP110102893. The contribution of Matthew Simpson was funded by the Australian Research Council's Discovery Project funding scheme, project number DP12000551.

\section{References}

[1] T. Alarcón, H. M. Byrne and P. K. Maini, "A cellular automaton model for tumour growth in inhomogeneous environment", J. Theoret. Biol. 225 (2003) 257-274;

doi:10.1016/S0022-5193(03)00244-3.

[2] R. M. Anderson and R. M. May, Infectious diseases of humans (Oxford University Press, Oxford, 1991).

[3] C. Beauchemin, J. Samuel and J. Tuszynski, "A simple cellular automaton model for influenza A viral infections", J. Theor. Biol. 232 (2005) 223-234; doi:10.1016/j.jtbi.2004.08.001.

[4] B. J. Binder and K. A. Landman, "Exclusion processes on a growing domain", J. Theor. Biol. 259 (2009) 541-551; doi:10.1016/j.jtbi.2009.04.025.

[5] B. J. Binder and K. A. Landman, "Quantifying evenly distributed states in exclusion and nonexclusion processes", Phys. Rev. E. 83 (2011) 041914; doi:10.1103/PhysRevE.83.041914.

[6] B. J. Binder, K. A. Landman, D. F. Newgreen, J. E. Simkin, Y. Takahashi and D. Zhang, "Spatial analysis of multi-species exclusion processes: application to neural crest cell migration in the embryonic gut", Bull. Math. Biol. 74 (2012) 474-490; doi:10.1007/s11538-011-9703-z.

[7] B. J. Binder, K. A. Landman, M. J. Simpson, M. Mariani and D. F. Newgreen, "Modeling proliferative tissue growth: a general approach and an avian case study", Phys. Rev. E. 78 (2008) 031912; doi:10.1103/PhysRevE.78.031912.

[8] G. V. Bobashev, M. D. Goedecke, F. Yu and J. M. Epstein, "A hybrid epidemic model: combining the advantages of agent-based and equation-based approaches", in: Proceedings of the 2007 Winter Simulation Conference (ed. S. Henderson), (IEEE, Washington DC, 2007), 1532-1537; doi:10.1109/WSC.2007.4419767. 
[9] D. Chowdhury, A. Schadschneider and K. Nishinari, "Physics of transport and traffic phenomena in biology: from molecular motors and cells to organisms", Phys. Life Rev. 2 (2005) 318-352; doi:10.1016/j.plrev.2005.09.001.

[10] C. A. Chung, T.-H. Lin, S.-D. Chen and H.-I. Huang, "Hybrid cellular automaton modeling of nutrient modulated cell growth in tissue engineering constructs", J. Theor. Biol. 262 (2010) 267-278; doi:10.1016/j.jtbi.2009.09.031.

[11] J. C. Dallon and H. G. Othmer, "A discrete cell model with adaptive signalling for aggregation of Dictyostelium discoideum”, Phil. Trans. R. Soc. Lond. B 352 (1997) 391-417; doi:10.1098/rstb.1997.0029.

[12] K. Dietz, "Epidemics and rumours: a survey", J. R. Stat. Soc. A 130 (1967) 505-528; doi:10.2307/2982521.

[13] P. J. Diggle, Statistical analysis of spatial point patterns (Academic Press, London, 1983).

[14] R. Erban and S. J. Chapman, "Time scale of random sequential adsorption", Phys. Rev. E 75 (2007) 041116; doi:10.1103/PhysRevE.75.041116.

[15] B. Franz and R. Erban, "Hybrid modelling of individual movement and collective behaviour", in: Dispersal, individual movement and spatial ecology: a mathematical perspective (eds M. Lewis, P. K. Maini and S. V. Petrovskii), (Springer) in press.

[16] Z. Guo, P. M. A. Sloot and J. C. Tay, "A hybrid agent-based approach for modeling microbiological systems", J. Theor. Biol. 255 (2008) 163-175; doi:10.1016/j.jtbi.2008.08.008.

[17] A. Heppenstall, A. Evans and M. Birkin, "Using hybrid agent-based systems to model spatiallyinfluenced retail markets”, J. Artificial Soc. Social Simulation 9 (2006) 2;

http://jasss.soc.surrey.ac.uk/9/3/2.html.

[18] R. I. Hickson, G. N. Mercer and K. M. Lokuge, "A metapopulation model of tuberculosis transmission with a case study from high to low burden areas", PLoS ONE 7 (2012) e34411; doi:10.1371/journal.pone.0034411.

[19] S. W. Jones, "The enhancement of mixing by chaotic advection", Phys. Fluids A 3 (1991) 1081-1086; doi:10.1063/1.858089.

[20] M. J. Keeling and P. Rohani, Modeling infectious diseases in humans and animals (Princeton University Press, Princeton, NJ, 2008).

[21] M. J. Keeling and J. V. Ross, "On methods for studying stochastic disease dynamics", J. R. Soc. Interface 5 (2008) 171-181; doi:10.1098/rsif.2007.1106.

[22] H. A. Kelly, G. N. Mercer, J. E. Fielding, G. K. Dowse, K. Glass, D. Carcione, K. A. Grant, P. V. Effler and R. A. Lester, "Pandemic (H1N1) 2009 influenza community transmission was established in one Australian state when the virus was first identified in North America", PLoS ONE 5 (2010) e11341; doi:10.1371/journal.pone.0011341.

[23] J. H. Phelps and C. L. Tucker, "Lagrangian particle calculations of distributive mixing: limitations and applications”, Chem. Engrg. Sci. 61 (2006) 6826-6836; doi:10.1016/j.ces.2006.07.008.

[24] M. G. Roberts, M. Baker, L. C. Jennings, G. Sertsou and N. Wilson, "A model for the spread and control of pandemic influenza in an isolated geographical region", J. R. Soc. Interface 4 (2007) 325-330; doi:10.1098/rsif.2006.0176.

[25] G. Rousseau, B. Giorgini, R. Livi and H. Chaté, "Dynamical phases in a cellular automaton model for epidemic propogation", Physica D 103 (1997) 554-563; doi:10.1016/S0167-2789(96)00285-0.

[26] J. Sewall, D. Wilkie and M. C. Lin, "Interactive hybrid simulation of large-scale traffic", ACM Trans. Graphics 30 (2011) Article No. 35; doi:10.1145/2070781.2024169.

[27] M. J. Simpson, K. A. Landman and B. D. Hughes, "Multi-species simple exclusion processes", Physica A 388 (2009) 399-406; doi:10.1016/j.physa.2008.10.038.

[28] M. J. Simpson, K. A. Landman and B. D. Hughes, "Cell invasion with proliferation mechanisms motivated by time-lapse data", Physica A 389 (2010) 3779-3790; doi:10.1016/j.physa.2010.05.020.

[29] M. J. Simpson, A. Merrifield, K. A. Landman and B. D. Hughes, "Simulating invasion with cellular automata: connecting cell-scale and population-scale properties", Phys. Rev. E 76 (2007) 021918; doi:10.1103/PhysRevE.76.021918. 
[30] J. Snow, On the mode of communication of cholera (John Churchill, London, 1854).

[31] S. Venkatachalam and A. R. Mikler, "An infectious disease outbreak simulator based on the cellular automata paradigm", in: Innovative internet community systems, Volume 3473 of Lecture Notes in Computer Science (eds T. Böhme, V. M. Larios Rosillo, H. Unger and H. Unger), (Springer, Berlin, 2006), 198-211.

[32] Z. Wang and T. S. Deisboeck, "Computational modeling of brain tumors: discrete, continuum or hybrid?", Sci. Model. Simul. 15 (2008) 381-393; doi:10.1007/s10820-008-9094-0.

[33] S. H. White, A. M. del Rey and G. Rodríguez Sánchez, "Modeling epidemics using cellular automata", Appl. Math. Comput. 186 (2007) 193-202; doi:10.1016/j.amc.2006.06.126. 